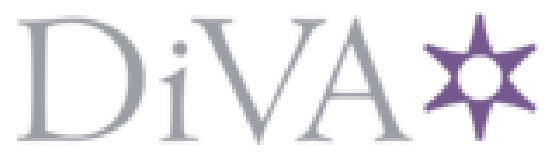

http://www.diva-portal.org

\title{
Postprint
}

This is the accepted version of a paper published in Journal of Surveying Engineering. This paper has been peer-reviewed but does not include the final publisher proof-corrections or journal pagination.

Citation for the original published paper (version of record):

Horemuz, M., Jansson, P. (2016)

Optimum Establishment of Total Station.

Journal of Surveying Engineering, 143(2)

https://doi.org/10.1061/(ASCE)SU.1943-5428.0000207

Access to the published version may require subscription.

N.B. When citing this work, cite the original published paper.

Permanent link to this version:

http://urn.kb.se/resolve?urn=urn:nbn:se:kth:diva-206173 


\section{Optimum establishment of total station}

Milan Horemuž, and Patric J ansson ${ }^{2}$

J . Surv. Eng. (2016), http://doi.org/ 10.1061/ (ASCE)SU.1943-5428.0000207

\section{Abstract}

At least two control points must be available in order to determine the position and orientation of a total station (TS). This paper analyses optimum horizontal location of TS with respect to the control points and gives an answer to question "What is the best location of total station with respect to the control points if the goal is to determine the coordinates of TS and detail points as precisely as possible?" The optimality is deemed based on the uncertainty of the horizontal coordinates of the TS and of the points measured from it, as well as on the uncertainty of the TS orientation. The investigation of this optimality problem was performed both analytically and by the trial-and-error method.

It was found that the optimum location of TS is in the center of gravity of all control points. For a given configuration of the control and detail points, the location of TS does not influence significantly the positional uncertainty of the surveyed detail points.

${ }^{1}$ Associate Professor, Dept. of Urban Planning and Environment, Div. of Geodesy and Satellite Positioning, KTH Royal Institute of Technology, SE-100 44 Stockholm, Sweden (corresponding author). E-mail: horemuz@kth.se

2 Ph.D., Dept. of Urban Planning and Environment, Div. of Geodesy and Satellite Positioning, KTH Royal Institute of Technology, SE-100 44 Stockholm, Sweden. E-mail: patricja@kth.se 


\section{Introduction}

In the majority of tasks where a total station (TS) is used as a surveying instrument, the goal is to determine or to set out coordinates of points in a required reference system. To achieve this goal, the TS must be georeferenced, i.e. the position and orientation of the TS in the required reference system must be established prior its use for detail surveying or staking out. There are two methods of TS establishment, i.e. the determination of its position and orientation: i) centering over a control point and orienting towards another control point(s) and ii) free station, i.e. measuring angles and distances to at least two control points (CPs). The same mathematical model can be applied for both methods and therefore the method i) can be considered as a special case of method ii).

Among surveyors it is well known that at least two CPs are required for station establishment and that we should use more CPs (at least 3) to secure the reliability and accuracy of the results. But it is not well known how to locate the TS with respect to a given configuration of CPs.

Only horizontal location is analyzed in this paper. The reason for this limitation is that in most of the applications it is not possible to choose the vertical position of the TS - it must be set up onto the existing surface. As optimality criteria we consider the horizontal uncertainty of TS, uncertainty in the measured detail points and the uncertainty in the orientation of TS. These uncertainties, as well as the error ellipses can be extracted from the covariance matrix of the unknown parameters.

The reliability is another important indicator of the goodness of TS setup. It can be divided into the internal and external reliability. The external reliability refers to the effect of the undetectable errors on the estimated parameters and the internal reliability refers to the ability to detect gross errors in the observations (see e.g. 
Ghilani 2010). The geometrical strength analysis (robustness analysis) is another aspect of reliability (Vaníček et al. 1990, 2001; Berber et al. 2006;). Seemkooei (2001a, b) showed that reliability and geometrical strength criteria are highly interrelated.

Finding the optimal location of TS setup belongs to the content of the classical geodetic problem of the optimal design of geodetic network.

The optimal design problem of geodetic networks originates from the works of Baarda (1973) and Grafarend (1974) who introduced four orders of design: zero, first, second, and third. The zero order design deals with the optimum choice of the datum, first order design finds the best geometrical configuration of the network, the second order design is about choosing the optimum weights, i.e. the precision of observations and the third order design deals with the densification of existing networks. The problem treated in this paper belongs to the category of the first order design.

When trying to locate the network points optimally the range of possibilities can vary considerably depending on the conditions in the working area. In some situation there is no room for choosing the distribution of the points because of physical constraints (terrain, buildings etc.) and the designer must place the points in a nonoptimal or sub-optimal way. But in other scenarios where any possible location within the area is acceptable, the best possible geometry should be chosen. There are two methods that can be used to find the best configuration of networks: analytical and trial-and-error. The analytical approach is based on a strict mathematical solution of the optimization problem, i.e. minimizing or maximizing a chosen objective function defined by the covariance matrix. Koch $(1982,1985)$ considered quadratic programming to optimize the configuration of a network. This approach 
was further developed by Kuang (1991, 1996), who considered different types of optimization methods, where the optimization procedure finds such geometry of the network, which gives the covariance matrix with smallest possible deviation from a chosen criterion matrix. In the trial-and-error approach, the covariance matrix is computed for a number of selected geometries. The geometry that fulfils the pre-set requirements or yields the best value of the objective function, is chosen as the best one.

It is also possible to specify more objective functions and find a multi-objective optimal solution as shown in Xu and Grafarend (1995). Bagherbandi et al. (2009) compared the single- and multi-objective optimization models in a simulated geodetic network and concluded that the single-objective model, which minimizes the reliability, is the best model for providing both high reliability and precision. As it will be shown in the following section, in the case of TS establishment the reliability does not depend on the location of TS, which is why the precision criterion is more suitable here. There are many publications on the optimization of geodetic networks; most of them consider optimization of larger networks but surprisingly very few of them analyse the simplest one, but very often used, i.e. the free stationing, which is resection using both angles and distances. We could find only three similar studies to the one presented in this paper: Amiri-Seemkooei et al. (2012), Sun (2013) and Horemuz and Andersson (2011). Amiri-Seemkooei et al. (2012) applied the analytical optimization method to solve the first order design problem for several cases of simple geodetic networks: traverse, resection with two and three distances and resection using three angles, but the paper does not treat the resection using both angles and distances. Sun (2013) examined the precision of free-station positioning, but only for the case with two control points and only by the trial-and-error method. 
However, due to the absence of orientation parameter in the observation model the conclusions in this study are not the same as ours.

Horemuz and Andersson (2011) performed precision analysis in free-station establishment by RTK GPS using trial-and-error approach. The main goal of this analysis was to find an optimum number and distribution of RTK GPS points, which serve as CPs for TS establishment. Uncertainties in the TS horizontal coordinates and in its orientation were chosen as the optimality criteria.

The goal of this paper is to analyze the precision of free-station establishment and, based on this analysis, formulate recommendations for the optimum placement of TS with respect to the $\mathrm{CP}$ in order to achieve the lowest possible uncertainty in the TS horizontal position, orientation, as well as in the horizontal position of DPs measured from the TS. The investigation of this optimality problem is performed both analytically and by the trial-and-error method.

The results of this study can be of interest to the geodetic community for many surveying engineering and land surveying applications. In addition, they can be instructive from the educational point of view.

\section{Observation model}

The TS is established if its coordinates and orientation is known in the required reference system. The establishment is achieved by measuring the distances and directions towards one or more CPs. The following observation model will be used in our analysis (see e.g. Kahmen, H. and Faig, W. (1988)):

$$
\begin{gathered}
\tilde{d}_{i}+v_{d i}=\sqrt{\left(N_{T S}-N_{i}\right)^{2}+\left(E_{T S}-E_{i}\right)^{2}} \\
\tilde{\Psi}_{i}+v_{\psi i}=\arctan \frac{E_{i}-E_{T S}}{N_{i}-N_{T S}}-r_{H}
\end{gathered}
$$




$$
\begin{gathered}
\tilde{E}_{i}+v_{E i}=E_{i} \\
\tilde{N}_{i}+v_{N i}=N_{i} \\
\tilde{d}_{D P}+v_{d D P}=\sqrt{\left(N_{T S}-N_{D P}\right)^{2}+\left(E_{T S}-E_{D P}\right)^{2}} \\
\tilde{\Psi}_{D P}+v_{\psi D P}=\arctan \frac{E_{D P}-E_{T S}}{N_{D P}-N_{T S}}-r_{H}
\end{gathered}
$$

where

$E_{\text {TS }}, N_{\text {TS }} \quad$ coordinates of TS

$\tilde{E}_{i}, \tilde{N}_{i} \quad$ measured (given) coordinates of CPs

$E_{i}, N_{i} \quad$ adjusted coordinates of common points $\mathrm{i}=1 . \mathrm{n}$

$\mathrm{n}$

number of CPs

$\mathrm{V}$

residuals, i.e. difference between the adjusted and measured quantity

$r_{H}$

orientation parameter, i.e. the angle between total station's zero direction and $\mathrm{N}$ axis.
$\tilde{d}$
measured horizontal distance
$\tilde{\Psi}$
measured horizontal direction
DP
detail point

After linearization of Eq. (1) and (2), we can write the observation model in matrix form as:

$$
\begin{gathered}
\underset{4 n \times 1}{\mathbf{L}}+\underset{4 n \times 1}{\mathbf{V}}=\underset{4 n \times 3}{\mathbf{A}} \mathbf{x} \mathbf{\mathbf { x }} \\
D(\mathbf{L})=E\left(\mathbf{v v}^{T}\right)=\mathbf{P}^{-1}
\end{gathered}
$$

where

L is vector of observations; there are 4 observations for each control point: two coordinates + one distance + one horizontal direction

$\mathbf{v}$ is vector of residuals 
A is design matrix

$\mathbf{x}$ is vector of parameters to be estimated

$\Delta \mathrm{E}_{T S}, \Delta N_{T S}, r_{H}, \Delta \mathrm{E}_{D P}, \Delta N_{D P}, \Delta \mathrm{E}_{C P 1}, \Delta N_{C P 1}, \ldots, \Delta \mathrm{E}_{C P n}, \Delta N_{C P n}$

$\mathrm{D}(\mathbf{L})$ is dispersion (covariance matrix) of observations

$\mathrm{E}$ is expectation operator

$\mathbf{P}$ is weight matrix of observations

The covariance matrix of the estimated parameters $\mathbf{x}$ :

$$
\mathbf{Q}_{X}=\left(\mathbf{A}^{\mathrm{T}} \mathbf{P A}\right)^{-1}
$$

The diagonal elements of $\mathbf{Q}_{\mathrm{x}}$ are variances of the adjusted parameters $\mathbf{x}$.

\section{Analytical approach}

In the first step, let us find such coordinates $E_{T S}, N_{T S}$, which yield minimum positional uncertainty $u(T S)=\sqrt{Q_{x 11}+Q_{x 22}}$. There is no elegant analytical solution if we apply the minimization problem to Eq. (8). It is easier to find such solution if the resection problem, i.e. Eq. (1) - (5), is reformulated as a 2D Helmert transformation. To do so, let us start with the equations relating the polar and Cartesian coordinates (Fig. 1):

$$
\begin{aligned}
& E_{i}=E_{T S}+d_{i} \sin \left(\psi+r_{H}\right) \\
& N_{i}=N_{T S}+d_{i} \cos \left(\psi+r_{H}\right)
\end{aligned}
$$

which can be rewritten

$$
\begin{aligned}
& E_{i}=E_{T S}+y_{i} \cos \left(r_{H}\right)+x_{i} \sin \left(r_{H}\right) \\
& N_{i}=N_{T S}+x_{i} \cos \left(r_{H}\right)-y_{i} \sin \left(r_{H}\right)
\end{aligned}
$$



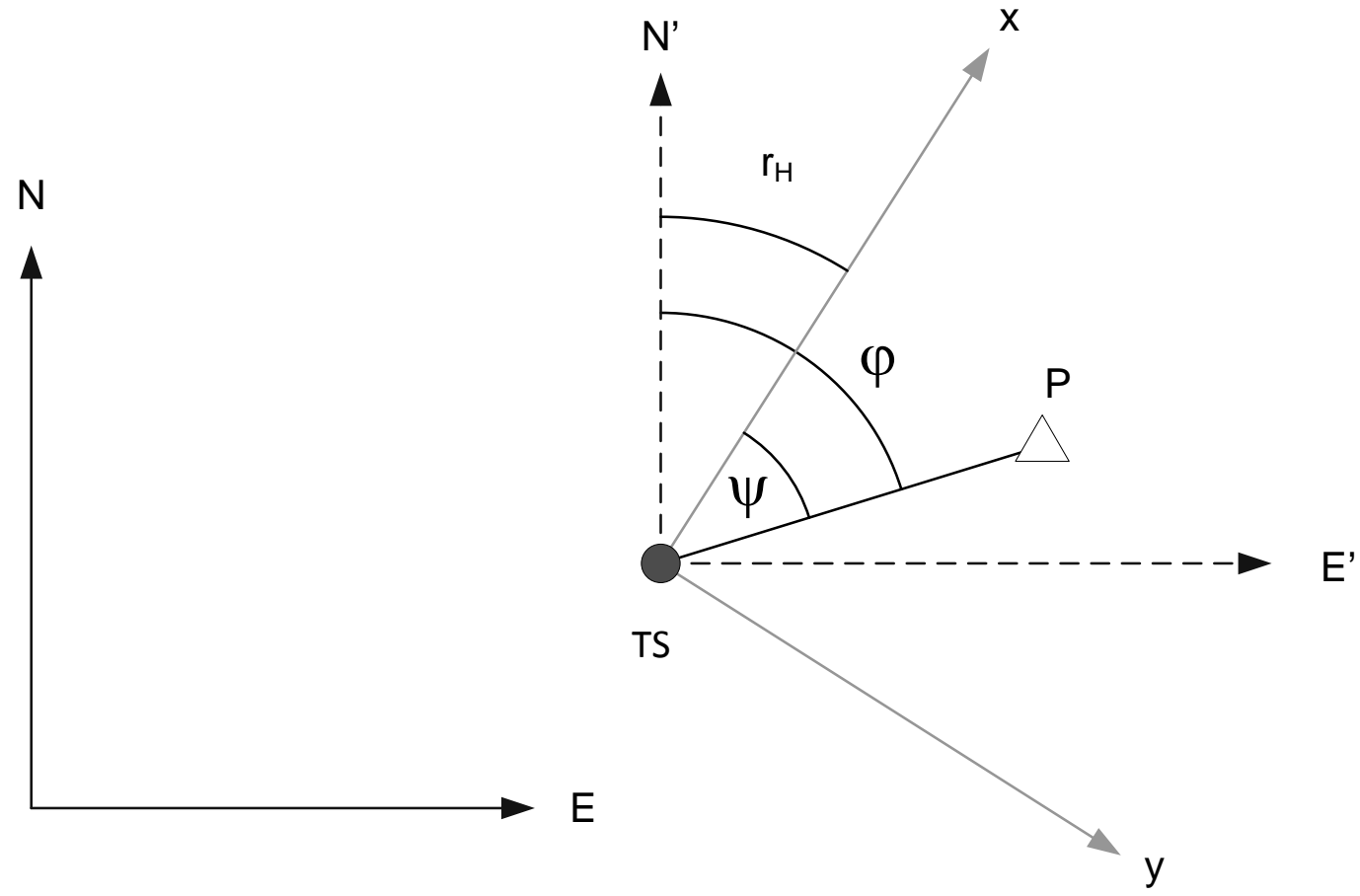

Fig. 1. Relation between external (NE) and total station (TS) coordinate system (xy).

These are well-known equations for 2D Helmert transformation (see e.g. Kahmen and Faig 1988), where $x_{i}=d_{i} \cos \psi$ and $y_{i}=d_{i} \sin \psi$ are the Cartesian coordinates of CP i in the TS coordinate system xy. Having at least two CPs, Eq. (10) can be linearized and solved by the least squares method. The linearization can be done by expansion using a Taylor series expansion around an approximate rotation angle $r_{H 0}$ : $r_{H}=r_{H 0}+\Delta r$ where $\Delta r$ is a correction to the approximate value. After the linearization, Eq. (10) becomes:

$$
\begin{aligned}
& E_{i}-y_{i} \cos r_{H 0}-x_{i} \sin r_{H 0}=E_{T S}+\left(x_{i} \cos r_{H 0}-y_{i} \sin r_{H 0}\right) \Delta r \\
& N_{i}-x_{i} \cos r_{H 0}+y_{i} \sin r_{H 0}=N_{T S}-\left(y_{i} \cos r_{H 0}+x_{i} \sin r_{H 0}\right) \Delta r
\end{aligned}
$$

or

$$
\begin{aligned}
& \tilde{E}_{i}-\tilde{E}_{i}^{\prime}+v_{E i}=E_{T S}+\left(x_{i} \cos r_{H 0}-y_{i} \sin r_{H 0}\right) \Delta r \\
& \tilde{N}_{i}-\tilde{N}_{i}^{\prime}+v_{N i}=N_{T S}-\left(y_{i} \cos r_{H 0}+x_{i} \sin r_{H 0}\right) \Delta r
\end{aligned}
$$


where "prime" denotes coordinates of control points determined by TS in system $\mathrm{N}^{\prime} \mathrm{E}^{\prime}$, which is xy system rotated so that it is parallel with NE system (see Fig. 1):

$$
\begin{aligned}
& E_{i}^{\prime}=y_{i} \cos r_{H 0}+x_{i} \sin r_{H 0}=d_{i} \sin \left(\psi+r_{H 0}\right) \\
& N^{\prime}=x_{i} \cos r_{H 0}-y_{i} \sin r_{H 0}=d_{i} \cos \left(\psi+r_{H 0}\right)
\end{aligned}
$$

Eq. (12) is now considered as the observation model, which can be written in matrix form (6), where

$$
\mathbf{L}=\left[\begin{array}{c}
E_{i}-E_{i}^{\prime} \\
N_{i}-N_{i}^{\prime}
\end{array}\right] ; \quad \mathbf{A}=\left[\begin{array}{ccc}
1 & 0 & x_{i} \cos r_{H 0}-y_{i} \sin r_{H 0} \\
0 & 1 & -\left(y_{i} \cos r_{H 0}+x_{i} \sin r_{H 0}\right)
\end{array}\right] ; \quad \mathbf{x}=\left[\begin{array}{c}
E_{T S} \\
N_{T S} \\
\Delta r
\end{array}\right]
$$

and the covariance matrix of the observations is

$$
\mathbf{P}^{-1}=\mathbf{Q}_{L}=E\left(\mathbf{v \mathbf { v } ^ { T }}\right)=\mathbf{Q}_{N E}+\mathbf{Q}_{N^{\prime} E^{\prime}}
$$

where $\mathbf{Q}_{N E}$ is the covariance matrix of coordinates of CPs in system NE and $\mathbf{Q}_{N^{\prime} E^{\prime}}$ the covariance matrix of coordinates of CPs determined by TS in system N'E'. It is assumed that all control points have the same positional uncertainty $\mathrm{u}(\mathrm{CP})$ with diagonal covariance matrix:

$$
\begin{aligned}
& \mathbf{Q}_{N E}=\frac{u^{2}(C P)}{2} \mathbf{I} \\
& u^{2}(C P)=u^{2}\left(E_{T S}\right)+u^{2}\left(N_{T S}\right)
\end{aligned}
$$

$\mathbf{Q}_{N^{\prime} E^{\prime}}$ can be determined by applying the law of variance propagation on equations (13):

$$
\mathbf{Q}_{N^{\prime} E^{\prime}}=\left[\begin{array}{ll}
d^{2} u^{2}(\Psi) \cos ^{2} \varphi_{0}+u^{2}(d) \sin ^{2} \varphi_{0} & \left(u^{2}(d)-d^{2} u^{2}(\Psi)\right) \sin \varphi_{0} \cos \varphi_{0} \\
\left(u^{2}(d)-d^{2} u^{2}(\Psi)\right) \sin \varphi_{0} \cos \varphi_{0} & d^{2} u^{2}(\Psi) \sin ^{2} \varphi_{0}+u^{2}(d) \cos ^{2} \varphi_{0}
\end{array}\right]
$$

where $\mathrm{d}$ is distance between TS and $\mathrm{CP}, \varphi_{0}=\psi+r_{H 0}$ (see Fig. 1) and $\mathrm{u}($ ) denotes standard uncertainty of the parameter in parentheses. For equal uncertainty in angular and distance measurements, i.e. if $d u(\Psi)=u(d)$, the off-diagonal elements of 
$\mathbf{Q}_{N^{\prime} E^{\prime}}$ become zero and the diagonal elements become equal. In this case $\mathbf{Q}_{L}=\left(u^{2}(N E)+u^{2}(d)\right) \mathbf{I}$ is a diagonal matrix.

If we choose to minimize the positional variance of TS

$$
u^{2}(T S)=u^{2}\left(E_{T S}\right)+u^{2}\left(N_{T S}\right)
$$

the optimum position can be determined by

$$
\left(E_{T S}, N_{T S}\right)_{O P T}=\underset{E_{T S}, N_{T S}}{\arg \min }\left(Q_{X 11}+Q_{X 22}\right)
$$

This equation has an elegant (= simple) solution if we consider equal uncertainty for all coordinate differences and zero covariance between them, i.e. $\mathbf{Q}_{\mathrm{L}}$ is a diagonal matrix with equal diagonal elements $\mathbf{Q}_{L}=\sigma_{0}^{2} \mathbf{I}$. In this case, the elements of $\mathbf{Q}_{\mathbf{x}}$ become:

$$
\begin{aligned}
& Q_{X 11}=\operatorname{var}\left(E_{T S}\right)=\frac{\sigma_{0}^{2}}{n D}\left[\sum_{i=1}^{n-1} \sum_{j=i+1}^{n}\left(E_{i}-E_{j}\right)^{2}+n \sum_{i=1}^{n}\left(N_{i}-N_{T S}\right)^{2}\right] \\
& Q_{X 22}=\operatorname{var}\left(N_{T S}\right)=\frac{\sigma_{0}^{2}}{n D}\left[\sum_{i=1}^{n-1} \sum_{j=i+1}^{n}\left(N_{i}-N_{j}\right)^{2}+n \sum_{i=1}^{n}\left(E_{i}-E_{T S}\right)^{2}\right] \\
& Q_{X 33}=\operatorname{var}\left(r_{H}\right)=\frac{n \sigma_{0}^{2}}{D} \\
& Q_{X 12}=\operatorname{cov}\left(E_{T S}, N_{T S}\right)=\frac{\sigma_{0}^{2}}{n D}\left(\sum_{i=1}^{n}\left(N_{i}\right)-n N_{T S}\right)\left(\sum_{i=1}^{n}\left(E_{i}\right)-n E_{T S}\right) \\
& D=\sum_{i=1}^{n-1} \sum_{j=i+1}^{n}\left(E_{i}-E_{j}\right)^{2}+\sum_{i=1}^{n-1} \sum_{j=i+1}^{n}\left(N_{i}-N_{j}\right)^{2}
\end{aligned}
$$

Eq. (20) was derived using the Symbolic Math Toolbox in Matlab. To solve Eq. (19), the following equations must be solved:

$$
\begin{aligned}
& \frac{\partial\left(\mathbf{Q}_{X 11}+\mathbf{Q}_{X 22}\right)}{\partial E_{T S}}=0 \\
& \frac{\partial\left(\mathbf{Q}_{X 11}+\mathbf{Q}_{X 22}\right)}{\partial N_{T S}}=0
\end{aligned}
$$

yielding: 


$$
E_{T S}=\frac{\sum_{i=1}^{n} E_{i}}{n} ; \quad N_{T S}=\frac{\sum_{i=1}^{n} N_{i}}{n} ;
$$

It can be easily shown that

$$
\begin{aligned}
& \frac{\partial^{2}\left(\mathbf{Q}_{X 11}+\mathbf{Q}_{X 22}\right)}{\left(\partial E_{T S}\right)^{2}}>0 \\
& \frac{\partial^{2}\left(\mathbf{Q}_{X 11}+\mathbf{Q}_{X 22}\right)}{\left(\partial N_{T S}\right)^{2}}>0
\end{aligned}
$$

which means that the solution (22) is the minimum of $\mathrm{Q}_{\mathrm{x} 11}+\mathrm{Q}_{\mathrm{x} 22}$. It means that the optimal location of TS is in the center of gravity of the CPs. Kahmen (1988) arrived at the same conclusion, even though without mathematical proof. As can be seen from Eq. (20), the variance of $r_{H}$ does not depend on the coordinates of TS, it depends only on the coordinates of the control points.

Please note that the solution to Eq. (21) is the same as Eq. (22) not only for $\mathbf{Q}_{L}=\sigma_{0}^{2} \mathbf{I}$, but even if $\mathbf{Q}_{\mathrm{L}}$ is a block-diagonal matrix with equal block-diagonal elements, i.e. if all points surveyed by the TS (both control and detail points) have non-circular error ellipses of the same size and orientation. With this assumption, the covariance matrix $\mathbf{Q}_{\mathrm{L}}$ will be the same for any location of TS with respect to the NE system, which means that Eq. (20) can be used for computation of positional uncertainty of any point having coordinates $\mathrm{E}_{\mathrm{TS}}, \mathrm{N}_{\mathrm{TS}}$, i.e. we can determine the positional uncertainty of detail points by substituting $E_{T S}, N_{T S}$ with $E_{D P}, N_{D P}$. The consequence of this fact is that the positional uncertainty of detail points does not depend on the position of TS, only on the configuration of the control and detail points.

We can also note that $\mathrm{Q}_{\mathrm{x} 12}$ becomes zero for the optimum location of TS, i.e. the uncertainty of the TS position is equal in all directions or in other words, the error ellipse has zero flattening, i.e. it is a circle. 
Reliability or robustness against the gross errors depends on the redundancy numbers $\left(r_{\mathrm{i}}\right)$ of individual observations, which can be found on the diagonal of the redundancy matrix $\mathbf{R}$ (see e.g. Leick 1995):

$$
\mathbf{R}=\mathbf{I}-\mathbf{A}\left(\mathbf{A}^{\mathrm{T}} \mathbf{P A}\right)^{-1} \mathbf{A}^{\mathrm{T}} \mathbf{P}
$$

The value of a redundancy number can be between zero and one. Zero redundancy means that any gross error will fully affect the results and the corresponding residual will always be zero and value 1 means that any gross error in that observation can be detected; the residual is the gross error itself. Ideally, all redundancy numbers should be equal and close to 1 . To achieve such ideal redundancies, many redundant observations with a suitable geometry are required, which increases the cost of the surveying project. A value of $r_{1}$ around 0.5 or greater is usually a good trade-off between the cost and internal reliability (Ghilani 2010). Assuming block diagonal matrix $\mathbf{Q}_{L}$, the redundancy numbers do not depend on the coordinates of TS, which means that the reliability of TS establishment does not depend on the location of TS, only on the number and geometry of CPs.

\section{Trial-and-error approach}

Another way of finding the best position of TS is to compute its positional uncertainty for different locations, which are regularly spread over the area of interest. The location yielding the least positional uncertainty $\mathrm{u}(\mathrm{TS})$ is then considered as the optimum one.

In the analytical approach we assumed a diagonal/block-diagonal $\mathbf{Q}_{\mathrm{L}}$ with equal diagonal/block-diagonal elements, but in practice, $\mathbf{Q}_{\mathrm{L}}$ depends on the distance $\mathrm{d}$ and the bearing $\varphi$, see Eq. (17). The trial-and error approach can be used to verify the 
results from the analytical approach and also to test the influence of the non-equality of diagonal elements of $\mathbf{Q}_{\mathrm{L}}$.

In the following numerical tests a $1 \times 1 \mathrm{~m}$ grid was used as the locations of the TS. For every location of the TS a covariance matrix was computed by Eq. (8) using various number and configuration of CPs. Our numerical tests using $\mathbf{Q}_{\mathrm{L}}$ with equal blockdiagonal elements confirmed the result from the analytical approach: the optimal location of total station was in the centre of gravity CPs for all tested configurations of CPs.

To test the configurations with non-equal block-diagonal elements of $\mathbf{Q}_{\mathrm{L}}$ we considered the following uncertainties: $u(d)=2 \mathrm{~mm}+2 \mathrm{ppm}, \mathrm{u}(\psi)=1.5$ mgon and $\mathrm{u}(\mathrm{CP})=10 \mathrm{~mm}$; hence $\mathbf{Q}_{\mathrm{L}}$ will be different for each test location and its blockdiagonal elements are not equal Fig. 2 shows the standard uncertainty in position and orientation of TS in the case of two control points. The location, which yields the least uncertainty in position and orientation, is depicted by red dots. In this case it is in the center of gravity of CPs since for this location, the covariance matrix $\mathbf{Q}_{\mathrm{L}}$ has equal block-diagonal elements. Fig. 2 shows also the shape of error ellipse and its flattening, which is computed as

$$
\text { flattening }=\frac{a-b}{a}
$$

where $\mathrm{a}$ and $\mathrm{b}$ is semi major and semi minor axis of the error ellipse. If the flattening is zero, then the ellipse becomes a circle. The least flattening does not always coincide with the optimal location of TS. This depends on the distribution of CPs as well as on the uncertainty of the observations. In this case there are two flattening minima, but it should be noted that the difference between the minimum flattening and the flattening at the optimum location of TS is negligible (0.04). One more case with a 
symmetric distribution of CPs is shown in Fig. 3, where four CPs form a square. The optimum location of TS is again in the center of gravity of CPs. We have tested several other symmetric cases with the same result: $\mathrm{u}(\mathrm{TS})$ is minimal in the center of gravity of CPs.
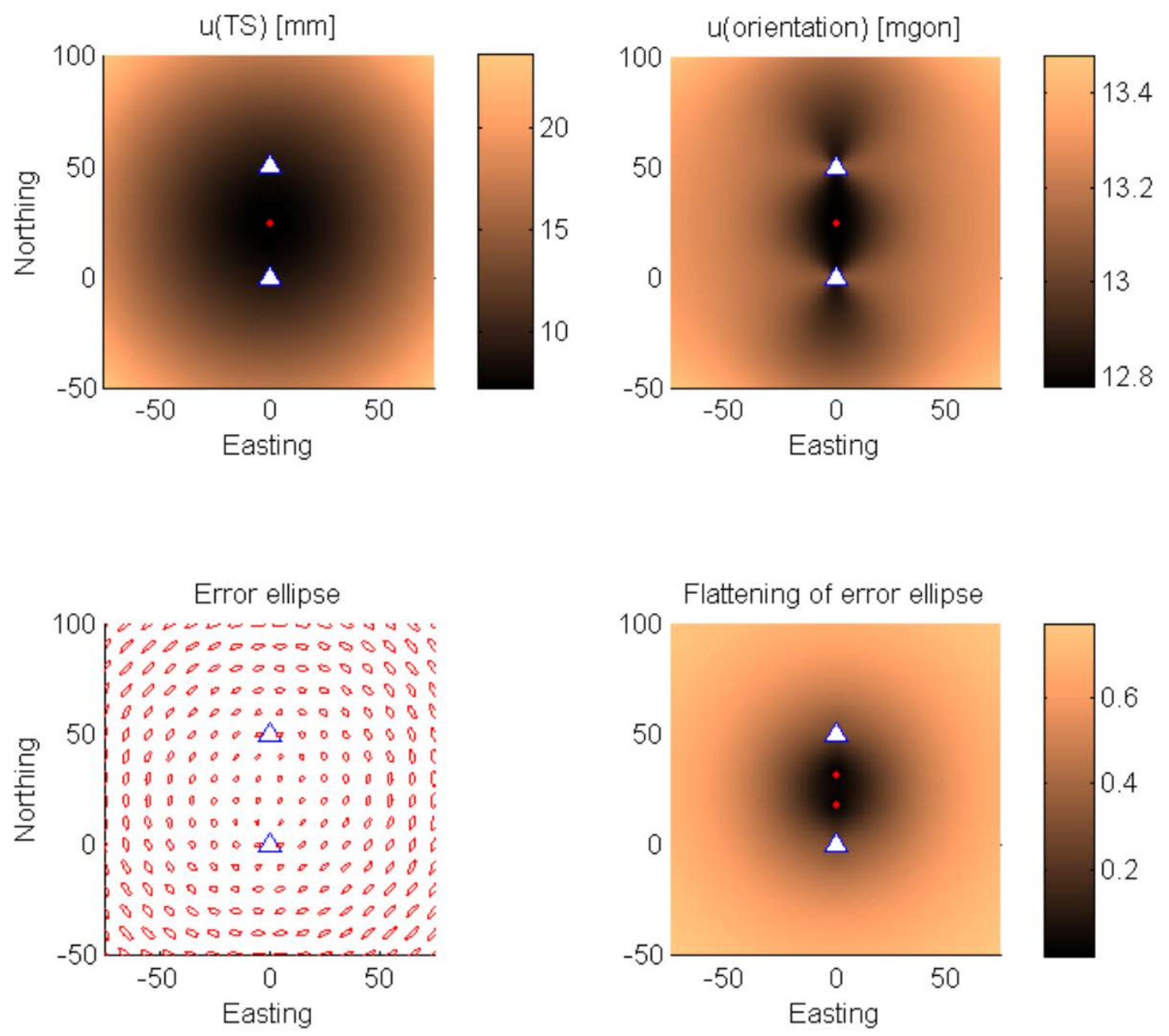

Fig. 2. Uncertainty in position and orientation of TS and shape of error ellipse. Two control points. Red dots depict the location, which yields the least values. 

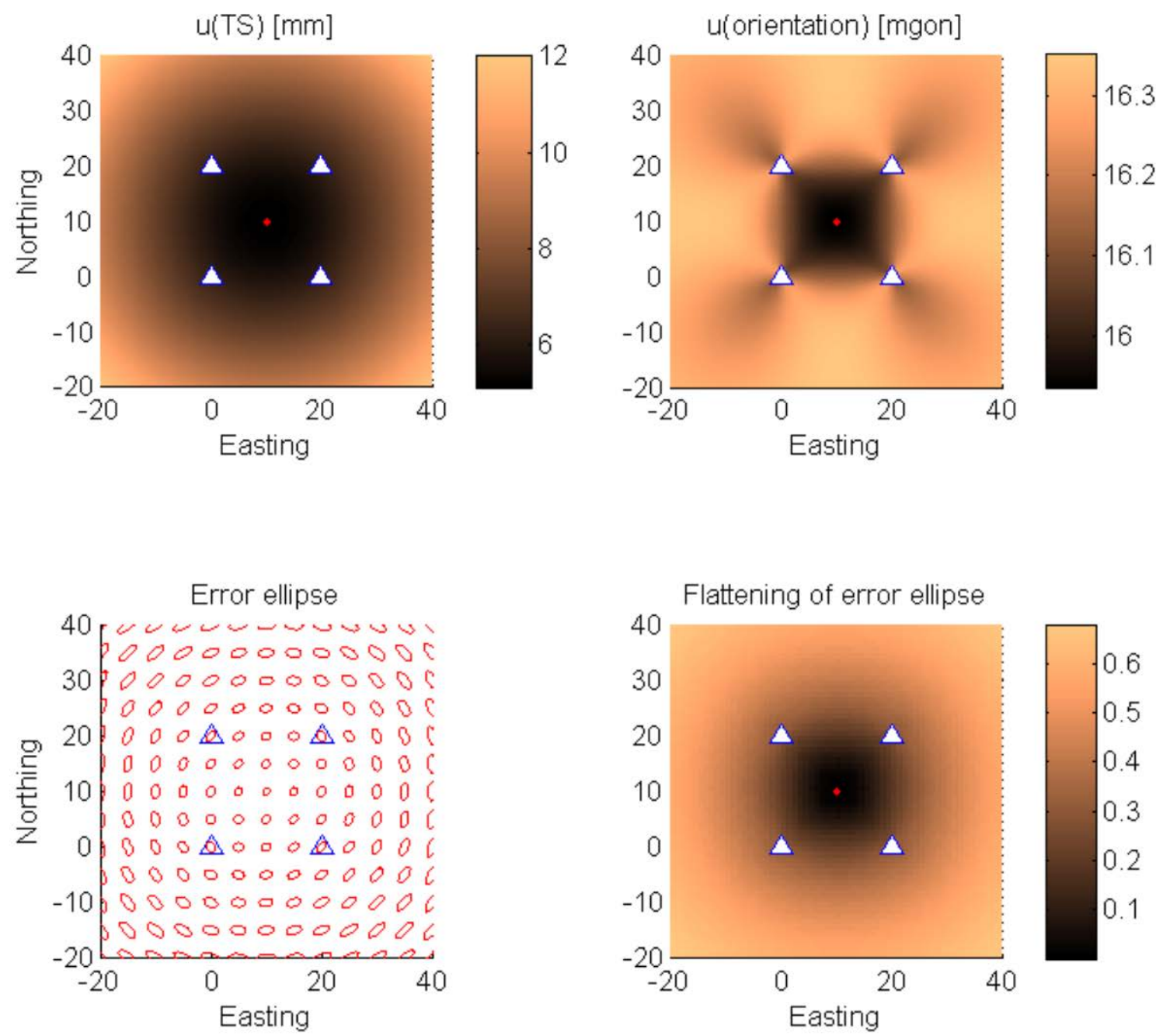

Fig. 3. Uncertainty in position and orientation of TS and shape of error ellipse. Four control points, symmetric distribution. Red dots depict the location, which yields the least values.

Fig. 4 shows an example of non-symmetric distribution of control points. None of red points is located in the center of gravity of CPs. The location of TS that yields the least $\mathrm{u}(\mathrm{TS})$ is located $1 \mathrm{~m}$ away from the center of gravity, u(orient) $10 \mathrm{~m}$ and least flattening $5 \mathrm{~m}$. However, the difference between $\mathrm{u}(\mathrm{TS}), \mathrm{u}$ (orient) and the flattening in the center of gravity and in the optimum location is negligibly small. 

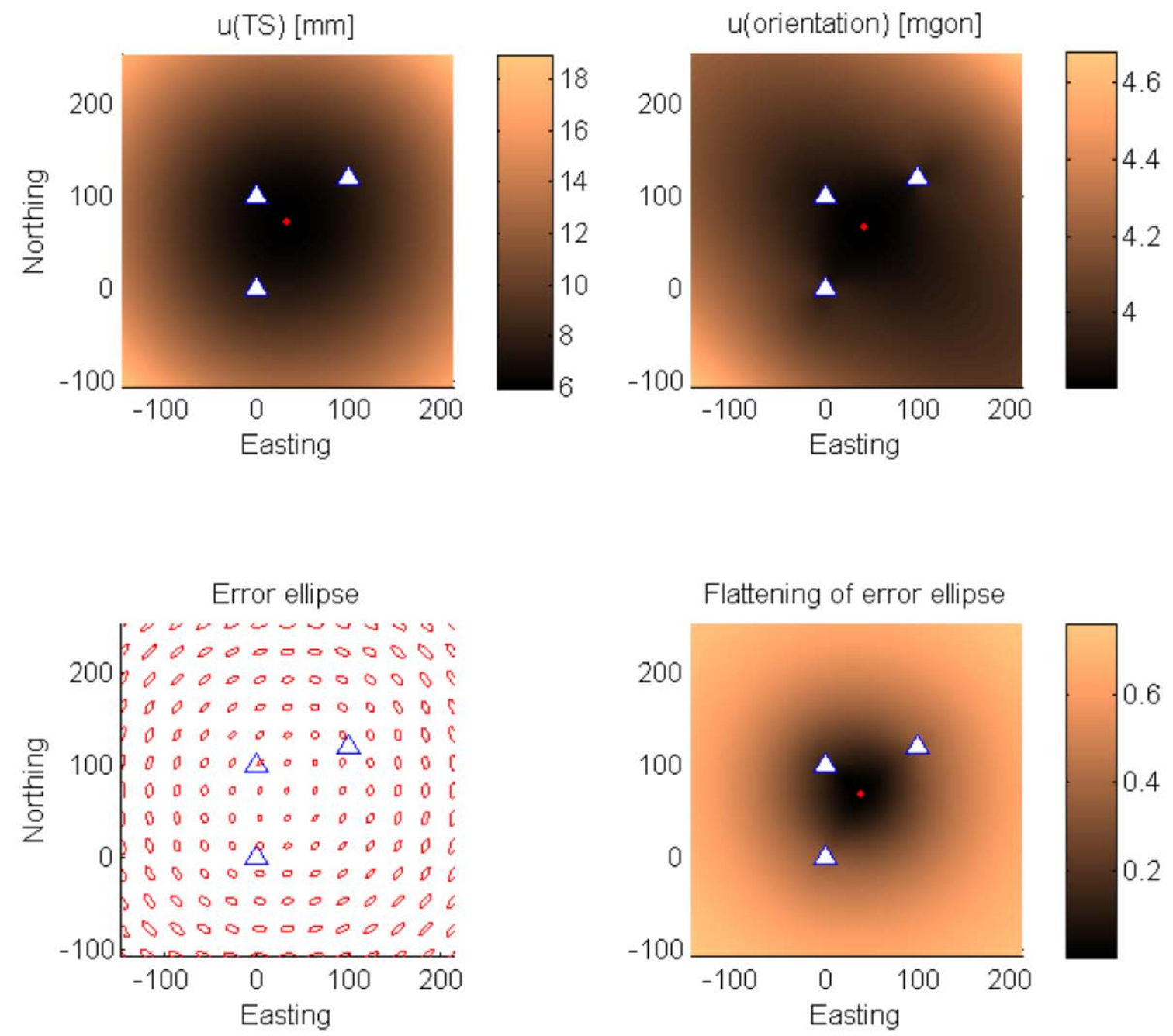

Fig. 4. Uncertainty in position and orientation of TS and shape of error ellipse. Three control points, non-symmetric distribution. Red dots depict the location, which yields the least values

In our numerical test we also computed the positional uncertainty $\mathrm{u}(\mathrm{DP})$ and flattening of the error ellipse of a detail point surveyed from the TS. According to the analytical method, $\mathrm{u}(\mathrm{DP})$ does not depend on the location of TS if $\mathbf{Q}_{\mathrm{L}}$ has equal diagonal/block-diagonal elements. Since this assumption did not hold in our numerical tests, $\mathrm{u}(\mathrm{DP})$ varied depending on the location of TS. As can be seen in Fig. 5 , the variation of $u(D P)$ and of flattening is very small. In the examples shown in this figure, the least $\mathrm{u}(\mathrm{DP})$ is obtained for location of TS on the DP (Fig. 5b and c) and for 
the location of TS on the nearest CP (Fig. $5 \mathrm{a}$ )). There is no simple general rule for the best location of TS with respect to $\mathrm{u}(\mathrm{DP})$, since it depends on the location of DP, number and distribution of CPs as well as on the uncertainties of the observations. But for all realistic scenarios, the variation of $u(D P)$ as well as flattening is negligibly small and hence the location of TS does not affect the results significantly.
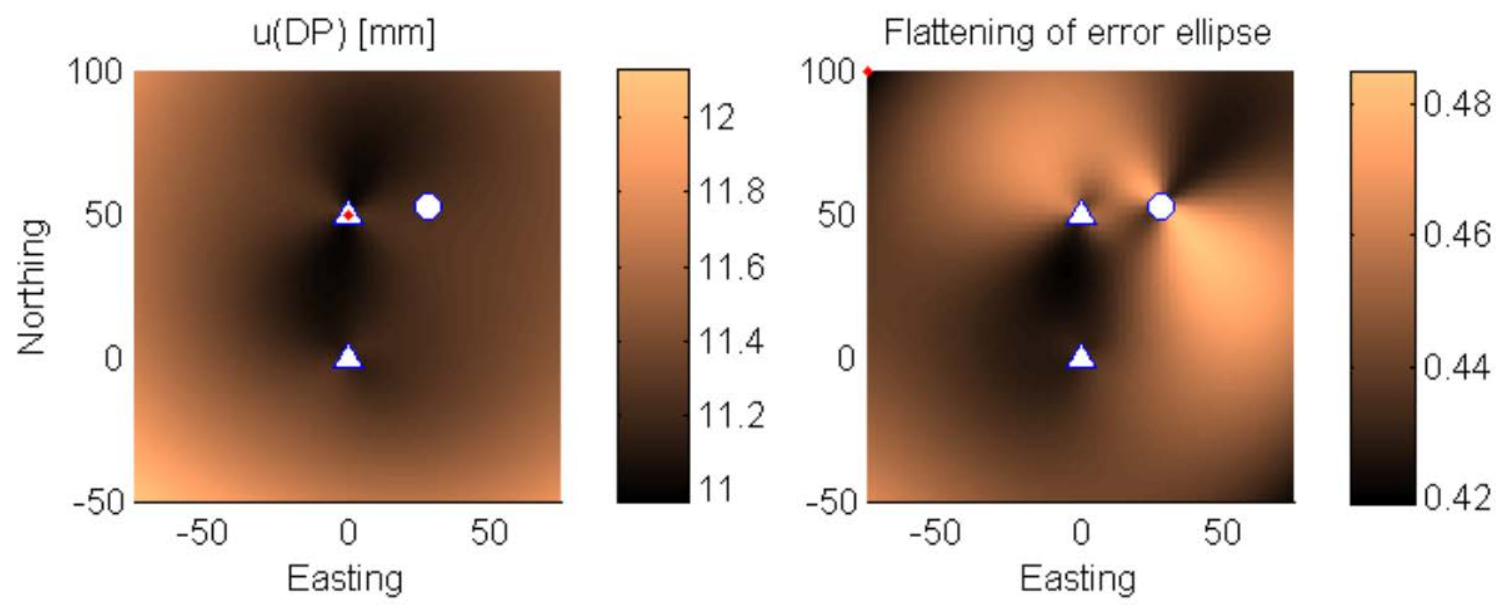

a)
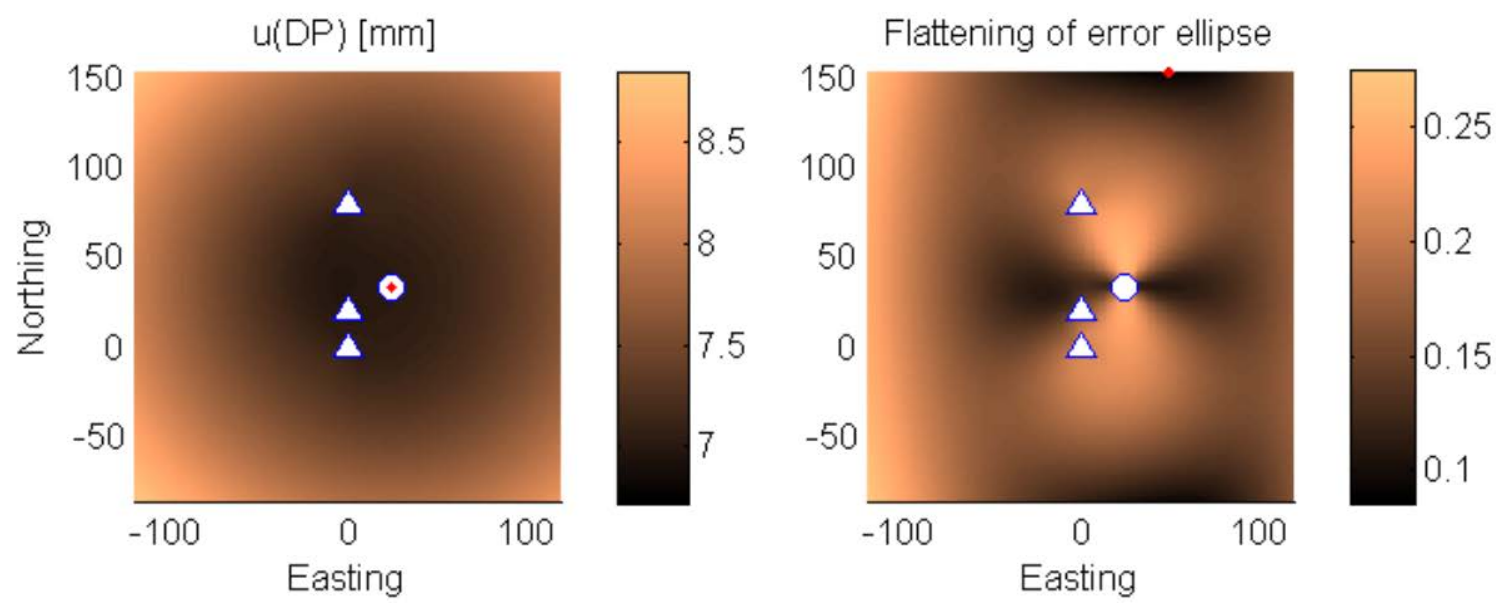

b) 

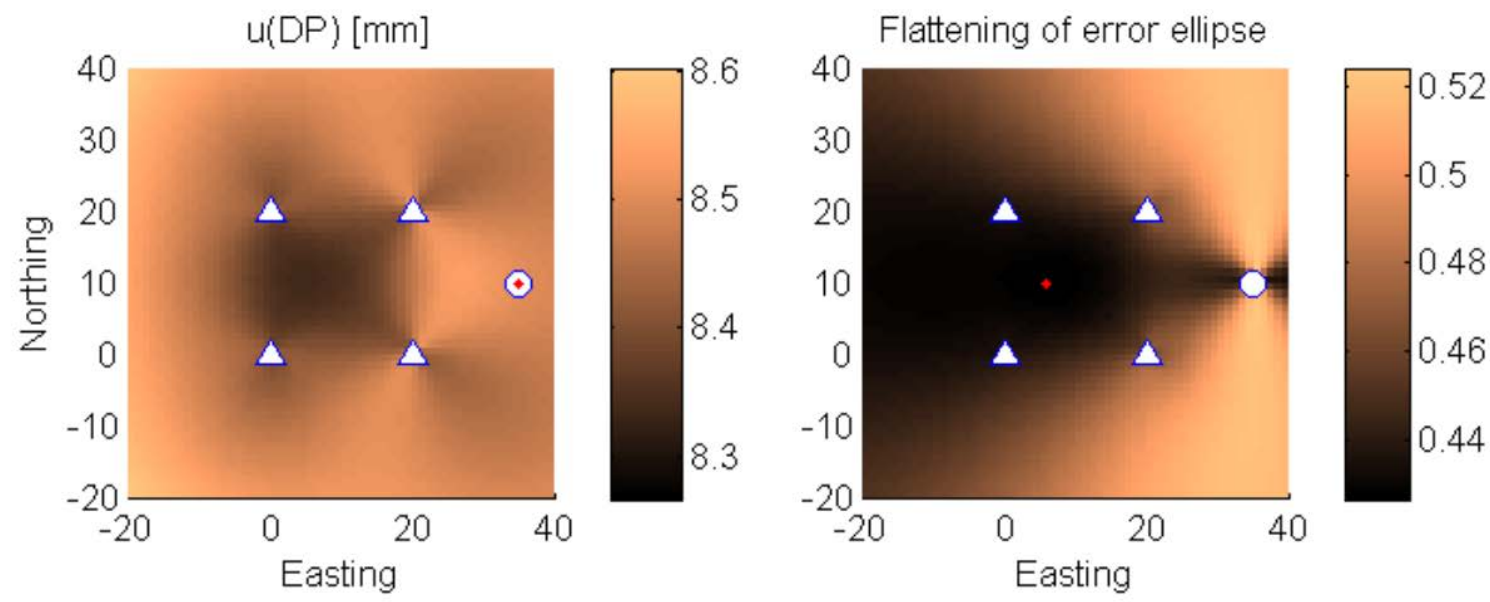

C)

Fig. 5. Uncertainty in position and flattening of error ellipse of a detail point (circle). a) 2 control points (triangles), b) 3 CPs, non-symmetric distribution, c) 4 CPs, symmetric distribution. Red dots depict the location, which yields the least values.

\section{Discussion and conclusions}

In this paper we analyzed the optimum horizontal location of TS with respect to the control points for establishment of free station. The analysis was done by analytical and by trial-and-error method. If we assume that all points surveyed by TS have equal positional uncertainty expressed in the instrument's coordinate system, then the position of TS in the center of gravity of all CPs yields the least uncertainty in the position of TS. The same conclusion can be found in classical surveying textbooks (e.g. Kahmen and Faig 1988) but without analytical proof. Even the flattening of the error ellipse is smallest for this location of TS. The uncertainty in orientation and the reliability of observations does not depend on the location of TS; it depends only on the number and distribution of the CPs. Another finding of our analysis is that the positional uncertainty of a DP measured from the free station does not depend on the 
location of TS; it only depends on the number and distribution of CPs.. The DP located in the center of gravity of CPs is determined with the least uncertainty. These conclusions were proven analytically and also verified by the trial-and-error method. In most of the practical applications, the points surveyed by TS do not have equal uncertainties. In this case there is no simple analytical solution for the placement of TS and therefore we applied the trial-and-error method. We showed on several cases that the optimum placement of TS does not always coincide with the center of gravity of CPs, but it depends on the configuration of CPs and the uncertainty of TS observations. However, the difference between the uncertainty in the orientation and horizontal position of TS in the optimum location and the center of gravity is negligible, if we consider approximate equality in uncertainty of angular and distance measurements, i.e. $d u(\Psi) \approx u(d)$, which is the case for most of the instruments. So for most of practical purposes one can consider the center of gravity of CPs as the best location of TS. This is an important conclusion, which is applicable if the free station method is used to establish new control point.

If we consider significantly smaller $(d u(\Psi)<<u(d)) /$ greater $(d u(\Psi)>>u(d))$ uncertainty in angular observations, then the problem is converted into resection using angles/distances only. These cases were analyzed by Amiri-Simkooei et al (2012).

But in the case when the free station is used only as a temporary station for detail surveying, the positional uncertainty of the surveyed detail points is more important than the uncertainty in TS establishment. As we proved analytically, the location of TS does not influence the uncertainty in horizontal position of detail points if we assume the homogenous uncertainty of TS observations. If this assumption is violated, then different locations of TS yield different uncertainties in the DP. However, the variation of uncertainties of DP is negligible so for most practical 
purposes any convenient location of TS is "the best one". This is an important conclusion for applications with a possibility to choose the location of CPs. For example, in many deformation monitoring tasks, the monitored points are surveyed from one location of TS, which is established as a free station in every measurement epoch by measuring distances and angles towards a number of stable CPs. In this case, the least uncertainty in DPs is achieved if the CPs are distributed around the monitored points rather than around the instrument station. Another example is establishment of TS using GNSS-RTK observations: the points used for station establishment should surround the working area.

Recommendations for practitioners:

- If the free station is used for establishment of a new CP, the best location of TS is in or near the center of gravity of CPs.

- If the free station is used for detail surveying, locate the TS at the most convenient place from where you see all points to be measured; the location of TS has negligible influence on the uncertainty of the DPs.

- The uncertainty in a DP depends on its location with respect to the CPs; the least uncertainty has a DP located in the center of gravity of CPs.

- If there is a possibility to choose the location of CPs, distribute them around the DPs rather than around TS.

\section{Acknowledgement}

The authors would like to acknowledge the support from The Swedish Research Council FORMAS, project number 245-2012 -356.

\section{References}


Amiri-Simkooei, A.R., Asgari, J., Zangeneh-Nejad, F., and Zaminpardaz, S. (2012). “Basic concepts of optimization and design of geodetic networks.” J. Surv. Eng., 138, 172-183.

Baarda, W. (1973). "S-transformation and criterion matrices." Netherland Geodetic Commission, Delft, Netherlands.

Berber, M., Dare, P., and Vaníček, P. (2006). “Robustness analysis of twodimensional networks." J . Surv. Eng., 132(4), 168-175.

Eshagh, M. (2005). “Optimization and design of geodetic networks.” Ph.D. study report in geodesy, Royal Institute of Technology, Division of Geodesy, Stockholm, Sweden.

Ghilani, C.D. (2010). "Adjustment Computations: Spatial Data Analysis." Fifth Edition, J ohn Wiley \& Sons, Inc.

Grafarend, E.W. (1974). “Optimization of geodetic networks.” Boll. Geod. Sci. Aff., 33(4), 351-406.

Grafarend, E. (1975). “Second Order Design of Geodetic Nets.” Z.Vermessungswesen, 100, 158- 168.

Horemuz M. and Andersson J . V. (2011). Analysis of the precision in free station establishment by RTK GPS. Survey Review, 43, 323, pp.679-686.

Kahmen, H. and Faig, W. (1988). “Surveying.” Walter de Gruyter, Berlin, New York. Koch, K.R. (1982). "Optimization of the configuration of geodetic networks." Deutsche Geodaetische Kommission, B, 258/III, 82- 89, Munich. Koch, K.R. (1985). ”First Order Design: Optimization of the configuration of a network by introducing small position changes." Optimization and design of geodetic networks, E.W. Grafarend and F. Sansó, eds., Springer, Berlin, 56- 73. 
Kuang, S.L. (1991). "Optimization and Design of Deformation Monitoring Scheme." Ph.D. dissertation, Technical Report No. 157, Dept. of Surveying Engineering, Univ. of New Brunswick, Canada.

Kuang, S.L. (1993). "Second Order Design: Shooting for Maximum Reliability." J. Surv. Eng., Vol 119, No. 3, USA.

Kuang, S.L. (1996). Geodetic Network Analysis and Optimal Design: Concepts and Applications, Ann Arbor Press, Chelsea, MI, USA.

Leick, A. (1995). GPS satellite surveying. Wiley \& Sons, New York, Chichester, Toronto, Brisbane, Singapore.

Seemkooei, A.A. (2001a). "Comparison of reliability and geometrical strength criteria in geodetic networks."J . Geod., Vol. 75, pp. 227- 233.

Seemkooei, A.A. (2001b). "Strategy for designing geodetic network with high reliability and geometrical strength criteria." J. Surv. Eng., 127, 104-117. Sun, H. (2013). "Precision Analysis of Free-station Positioning in Total Station." Adv. Mat. Res., Vols. 694-697, 1281-1285.

Teunissen, P.J .G. (1985). “'Zero Order Design: Generalized inverses, Adjustment, the Datum problem and S-transformation." Optimization and design of geodetic networks, E.W. Grafarend and F. Sansó, eds., Springer, Berlin, 11- 55. Vaníček, P., Craymer, M.R. , and Krakiwsky, E.J . (2001). “Robustness analysis of geodetic horizontal networks."J . Geod., 75, 199-209.

Vaníček, P., Krakiwsky, E.J., Craymer, M.R., Gao, Y., and Ong, P.S. (1990). “Robustness analysis.” Tech. Rep. 156, Dept of Surveying Engineering, Univ. of New Brunswick, Canada.

Xu, P., and Grafarend, E. W. (1995). "A Multi-objective Second-Order Optimal Design for Deforming Networks." Geoph. J ourn. Int., 120(3), 577-589. 
